\title{
Evaluación del Xpert MTB RIF para el diagnóstico de tuberculosis y detección de resistencia a rifampicina en grupos vulnerables
}

\section{Evaluation of the Xpert MTB RIF for tuberculosis diagnosis and detection of rifampicin resistance in vulnerable groups}

\author{
María Rosarys Martínez-Romero, ${ }^{*}$ Tunyth Secretário-Chilemo, ${ }^{\ddagger}$ Dihadenys Lemus-Molina, ${ }^{*}$ \\ Lilian María Mederos-Cuervo, ${ }^{*}$ Misleidis Sardiñas-Aragón,* Grechen García-León,* \\ Miguel Echemendía-Font,* Raúl Díaz-Rodríguez*
}

*Laboratorio Nacional de Referencia e Investigaciones de Tuberculosis, Lepra y Micobacterias. Instituto de Medicina Tropical «Pedro Kourí». La Habana, Cuba; ${ }^{\ddagger}$ Licenciado en análisis clínico.

Director del Centro de Diagnóstico y Tratamiento de la Tuberculosis, Ondjiva, Angola.

\begin{abstract}
RESUMEN. Introducción: La emergencia de la tuberculosis y de cepas multidrorresistentes representa un problema para el control de la tuberculosis. El objetivo fue evaluar el Xpert MTB/RIF en grupos vulnerables. Material y métodos: Se estudiaron 82 muestras pulmonares con indicación de Xpert, en el Laboratorio Nacional de Referencia del Instituto «Pedro Kourí» de mayo a octubre del 2016. Se realizó la baciloscopia y cultivo según los procedimientos del laboratorio, el Xpert de acuerdo con las normas del fabricante. Los resultados del ensayo molecular se compararon con el cultivo (detección de Mycobacterium tuberculosis) y método de las proporciones (susceptibilidad a rifampicina). Resultados: Los grupos vulnerables donde más se diagnosticaron casos de tuberculosis fueron los VIH positivos (nueve), seguido de los previamente tratados (ocho). El Xpert detectó tuberculosis en 21 casos, de los cuales ocho fueron baciloscopia negativa. Existió buena concordancia entre Xpert y los métodos de cultivo (mayor de 92\%) y excelente coincidencia con el método de las proporciones. Conclusiones: El Xpert tuvo un efecto positivo en el manejo oportuno de casos. La excelente correlación entre Xpert MTB/RIF y el método de las proporciones demuestra la utilidad del sistema para la evaluación rápida de la resistencia a rifampicina como indicador de tuberculosis-multidrogorresistente.
\end{abstract}

Palabras clave: Tuberculosis, Mycobacterium tuberculosis, Xpert MTB/ RIF, rifampicina.

\begin{abstract}
Introduction: The emergence of tuberculosis (TB) and multidroresistant strains represents a problem for TB control. The objective was to evaluate the Xpert MTB/RIF (Xpert) in vulnerable groups (VG). Material and methods: We studied 82 pulmonary samples with Xpert indication, at the National Reference Laboratory, «Pedro Kourí» Institute, from May to October 2016. Sputum smear (AFB) and culture was performed, according to the laboratory procedures and Xpert according to the manufacturer's standards. The results of the molecular assay were compared with the culture (detection of Mycobacterium tuberculosis) and proportions method (susceptibility to rifampicin). Results: The VG where most cases of TB were diagnosed was HIV positive (nine), followed by those previously treated (eight). Xpert detected TB in 21 cases, of which, eight was AFB negative. There was good agreement between Xpert and culture methods (greater than 92\%) and excellent coincidence with the proportions method. Conclusions: The Xpert had a positive impact on the timely handling of cases. The excellent correlation between Xpert MTB/RIF and the proportions method demonstrates the utility of the system for the rapid assessment of rifampicin resistance as an indicator of TB - MDR.
\end{abstract}

Keywords: Tuberculosis, Mycobacterium tuberculosis, Xpert Mtb/RIF, rifampicin.

\section{INTRODUCCIÓN}

La tuberculosis (TB), es una de las 10 principales causas de muerte y la causa principal de un solo agente infeccioso (por encima del VIH/sida). La TB constituye un importante problema de salud a nivel mundial, principalmente en países de medianos y bajos ingresos. ${ }^{1}$ La emergencia y diseminación de cepas de Mycobacterium tuberculosis (Mtb) resistentes a diferentes drogas representa en la actualidad 
un serio problema para el control mundial de la TB, por lo que el diagnóstico temprano de una TB activa y la detección precoz de cepas multidrogorresistente (MDR) es esencial para interrumpir la cadena de transmisión. ${ }^{2}$

La principal limitación de los Programas de Control (PNC) de la TB es la dificultad para realizar el diagnóstico temprano de la TB. La baciloscopia ha sido el método inicial para el diagnóstico de la TB por su simplicidad, rapidez de procesamiento y bajo costo. Sin embargo, su baja sensibilidad (45-80\%), limita la utilidad de esta técnica. El cultivo es más sensible, pero requiere de técnicas más complejas, que demoran entre 30 y 60 días en demostrar el desarrollo micobacteriano. Por eso, en los últimos decenios se han desarrollado nuevos métodos de diagnóstico de la TB que intentan superar las limitaciones de la baciloscopia y del cultivo. $^{2-4}$

En la última década se han desarrollado técnicas moleculares para el diagnóstico de la TB que ofrecen mayor rapidez, con una buena sensibilidad y especificidad. Uno de estos sistemas es el ensayo Xpert, el cual fue recomendado por la Organización Mundial de la Salud (OMS) en el 2011. Tiene como ventaja que no requiere de una infraestructura especializada ni de personal altamente calificado, resulta menos complejo que otras pruebas moleculares, reduce el riesgo de contaminación cruzada y del personal del laboratorio, además, posibilita realizar el diagnóstico de $M t b$ de manera directa de una muestra clínica en menos de dos horas. ${ }^{5-7}$

La detección de resistencia a rifampicina (RIF), droga de primera línea en el tratamiento de la TB y a su vez marcadora de TB-MDR en países de alta carga, es de vital importancia. El principal mecanismo de resistencia de Mtb a esta droga está dado por mutaciones en el gen rpoB. Más del $90 \%$ de las cepas con resistencia a este fármaco muestran, además, resistencia a isoniacida, por lo tanto las personas pueden ser portadores de TB-MDR. Según la literatura, la sensibilidad para detectar la resistencia a la RIF con este método es alrededor de $95 \%$, pero con una especificidad menor. ${ }^{8}$

En Cuba la TB no constituye un problema de salud pública. En el año 2017 se notificaron un total de 659 casos para una tasa de notificación de $5.9 \times 100,000$ habitantes. ${ }^{9}$ Además, la vigilancia continua de la resistencia a las drogas antituberculosas indica que las cifras de TB-MDR en el país son bajas. ${ }^{10}$ Sin embargo, la coinfección TB/VIH ha mostrado una tendencia ascendente en los últimos años (12.3\% en el año 2014) (Ministerio de Salud Pública [MINSAP]; datos no publicados, 2016).

Para el Laboratorio Nacional de Referencia e Investigaciones de Tuberculosis, Lepra y Micobacterias (LNRI-TBLM) y el PNC de Cuba constituye una prioridad contar con herramientas que permitan un diagnóstico oportuno, tanto de la TB como de la resistencia a las drogas.

Recién el LNRI-TBLM dispone del Xpert. El PNC junto al LNRI-TBLM elaboró normativas basadas en las reco- mendaciones de la OMS, dirigidas a los grupos vulnerables (GV) con más probabilidad de contraer TB y desarrollar resistencia a las drogas antituberculosas. El empleo del ensayo molecular en GV, como los pacientes infectados por el VIH-sida, pudiera repercutir de manera positiva en el diagnóstico oportuno de la TB, ya que su mayoría cursa con baciloscopia negativa, así como en los casos de pacientes reclusos y exreclusos. Adicionalmente, permitiría detectar de forma rápida la resistencia a RIF en personal que realiza intercambio con países con elevada carga de TB resistente. Estas acciones contribuirán a un mejor control, diagnóstico y tratamiento de la TB, así como la vigilancia de la resistencia en Cuba. El objetivo del estudio fue evaluar las repercusiones del método Xpert MTB/RIF en GV.

\section{MATERIAL Y MÉTODOS}

Se realizó un estudio observacional, descriptivo, prospectivo de corte transversal. El universo estuvo conformado por todas las muestras pulmonares de pacientes con sospecha clínica de TB con indicación de Xpert recibidas en el LNRITBL-Instituto «Pedro Kourí», durante el período de mayo a octubre del 2016.

El LNRITBL-Instituto «Pedro Kourí», según las normativas elaboradas por el PNC, da prioridad para realizar el ensayo molecular a personas con mayor riesgo de enfermar de TB y desarrollar resistencia a las drogas como: contacto de casos de TB pulmonar bacilo ácido alcohol resistente (BAAR) positivo, pacientes $\mathrm{VIH}$, pacientes previamente tratados, reclusos y exreclusos, personal procedentes de países de alta carga de TB y TB-MDR, incluidos extranjeros, niños, individuos con otras enfermedades crónicas con alta sospecha de TB, trabajadores del sector salud relacionados con la atención a enfermos, así como personas de unidades de salud con internamiento prolongado (hogares de ancianos e impedidos físicos), por lo que el tamaño de la muestra quedó constituida por 82 especímenes.

Criterios de inclusión: muestras pulmonares con indicación de Xpert, identificadas de forma adecuada, con volumen igual o superior a $3 \mathrm{~mL}$ o a las que no se realizó el cultivo bacteriológico.

Criterios de exclusión: muestras derramadas, las que no estuvieran identificadas adecuadamente, volumen inferior a $3 \mathrm{~mL}$ o a las que no se realizó el cultivo bacteriológico.

Las muestras fueron divididas en 2 alícuotas: 1 mililitro $(\mathrm{mL})$ se procesó mediante el Xpert MTB/RIF; y al resto se le practicó la baciloscopia y procesamiento para el cultivo sólido y líquido, según lo establecido en el PNC de la TB. ${ }^{11}$

Procesamiento para el Xpert: se adicionó $2 \mathrm{~mL}$ del reactivo de muestra del Xpert MTB/RIF a $1 \mathrm{~mL}$ de esputo fresco. Posteriormente, se dio vórtex por unos segundos y se dejó actuar por 10 minutos, luego se mezcló por vórtex de nuevo y se esperó cinco minutos más. El esputo tratado 
se transfirió con pipeta estéril ( $2 \mathrm{~mL}$ ) al cartucho Xpert MTB/ RIF, se cargó al equipo y se esperó 1 hora y 50 minutos para la salida de los resultados de la prueba. La interpretación de los resultados se realizó de acuerdo al fabricante. ${ }^{12}$

Procesamiento para cultivo: las muestras pulmonares se descontaminaron por el método de Petroff modificado, para eliminar la flora normal. La inoculación, incubación y lectura se realizó, según lo establecido en el Procedimientos Normados de Operación (PNO) del laboratorio y el PNC ${ }^{11}$ de Cuba.

Identificación en especie: se realizó por el ensayo inmunocromatigráfico SD BIOLINE, para la detección del AgMPT64: antígeno MPT 64 (presente sólo en el complejo Mtb).

A todos los aislados identificados como complejo $M t b$, se les practicó el estudio de susceptibilidad a la rifampicina (RIF) por los métodos fenotípicos, según lo establecido en el PNO del laboratorio y el PNC.11

Análisis estadístico de los resultados y cálculo de los parámetros de desempeño del ensayo molecular: se utilizó el programa para análisis epidemiológico de datos tabulados EpiData (por sus siglas en inglés), versión 3.1 (EpiData Association, Dinamarca), con un intervalo de confianza del 95\%. Los resultados obtenidos por Xpert se compararon con las técnicas convencionales en cultivo sólido LJ. Los resultados de susceptibilidad a la RIF se compararon con los obtenidos mediante el método de las proporciones. Para calcular los indicadores de desempeño se utilizó como método de referencia el cultivo bacteriológico.

Aspectos éticos: no hubo conflictos de intereses entre los autores. El protocolo de investigación fue evaluado y aprobado por la Comisión Científica Especializada de Microbiología y el Comité de Ética de Investigación-IPK (Código de aprobación: CEI-IPK 50-16). Por el tipo de estudio que se realizó no se requirió de consentimiento informado de los pacientes que intervinieron en el estudio.

\section{RESULTADOS}

Por el Xpert MTB/RIF se detectó ADN de Mtb en 21 casos, y por el cultivo bacteriológico para micobacterias se identificó 15 aislados de $M t b$, seis casos menos que por el ensayo molecular.

En la tabla 1 se exponen los resultados obtenidos empleando el ensayo molecular Xpert MTB/RIF según los GV estudiados. Se debe señalar que el mayor número de muestras estudiadas pertenecieron a pacientes $\mathrm{VIH} /$ sida con 51 $(62.2 \%)$ y en nueve (17.6\%) se detectó la presencia de $M t b$, y dentro de ellos, cuatro fueron negativos a la baciloscopia. Es válido señalar que dentro de este grupo, cinco cumplían también la condición de haber sido reclusos o exreclusos. El segundo grupo que más casos de TB aportó fueron los previamente tratados (ocho), en todos se detectó $M t b$, dos de los cuales fueron baciloscopia negativa.

Xpert MTB/RIF detectó ADN de Mtb en 21 (25.6\%) casos, de éstos sólo en uno se detectó resistencia a la RIF, la prueba resultó negativa a TB en 57 (69.5\%) casos y en cuatro (4.9\%) se observó errores de la prueba o el resultado fue no válido.

También se compararon los resultados del Xpert con el cultivo. Para este análisis no se tuvieron en cuenta los cultivos contaminados o los resultados invalidados o con error al correr la prueba por el ensayo molecular. De los 21 casos positivos a Mtb por Xpert, se confirmaron 15 (71.4\%) por el cultivo y en seis $(28.6 \%)$ no se obtuvo crecimiento. Por otro lado, el Xpert MTB/RIF permitió detectar TB en ocho casos que habían sido negativos a la baciloscopia. Uno de los casos con baciloscopia positiva con resultados de TB no detectada por el Xpert resultó ser una micobacteria no tuberculosa por el cultivo.

Los indicadores de desempeño (ID) del Xpert MTB/ RIF para los casos con baciloscopia negativa, evidenció valores de sensibilidad (S), especificidad (E), concordancia (C), valor

Tabla 1: Resultados de Xpert MTB/RIF según los grupos vulnerables estudiados.

\begin{tabular}{|c|c|c|c|c|c|}
\hline \multirow[b]{2}{*}{ Grupos vulnerables } & \multirow[b]{2}{*}{ No. } & \multirow[b]{2}{*}{$\%$} & \multicolumn{3}{|c|}{ Casos positivos } \\
\hline & & & No. & Baciloscopia (+) & Baciloscopia (-) \\
\hline Previamente tratados & $8 / 82$ & 9.8 & 8 & 6 & 2 \\
\hline Extranjeros & $8 / 82$ & 9.8 & 1 & 0 & 1 \\
\hline Otras enfermedades crónicas & $5 / 82$ & 6.1 & 2 & 1 & 1 \\
\hline Hogar de ancianos & $3 / 82$ & 3.7 & 0 & 0 & 0 \\
\hline Fumadores & $2 / 82$ & 2.4 & 0 & 0 & 0 \\
\hline Trabajador salud & $1 / 82$ & 1.2 & 0 & 0 & 0 \\
\hline
\end{tabular}


predictivo positivo (VPP), valor predictivo negativo (VPN) de $100 \%, 92.86 \%, 93.22 \%, 42.86 \%$ y $100 \%$, respectivamente, con un índice de Youden muy cercano a 1 (0.93) (mayor de 0.75 para considerar una prueba diagnóstica aceptable). Para los casos BK (+), todos los ID de la prueba fueron de 100\% con un índice de Youden perfecto (Tabla 2).

La prueba de susceptibilidad a la RIF por el método de las proporciones se realizó a los aislados de Mtb con un número de colonias $\geq 10$, como resultado se obtuvo 15 aislamientos de Mtb sensibles a RIF, sólo uno resistente a esta droga. No se calcularon los indicadores de desempeño del ensayo molecular Xpert debido al escaso número de aislados estudiados, lo cual fue una limitante de este estudio.

\section{DISCUSIÓN}

Una de las principales prioridades de PNC a nivel mundial es la detección oportuna de los casos de TB y de la resistencia de TB-MDR. El diagnóstico rápido de la TB, es un paso importante para comenzar el tratamiento adecuado, implementar medidas y lograr un control epidemiológico efectivo de la enfermedad. ${ }^{13}$

Cuba muestra una baja tasa de incidencia de TB y se encuentra entre los países que pueden eliminar la enfermedad como problema de salud. Dentro de las tareas del Ministerio de Salud Pública de Cuba para lograr este propósito se encuentra la introducción de herramientas para el diagnóstico rápido de la TB, con prioridad para los GV, los cuales tienen de 30-50 veces más probabilidad de enfermar de TB que la población general. ${ }^{14}$

Una de las herramientas para lograr este fin es el sistema Xpert MTB/RIF, técnica molecular recomendada por la OMS en el 2011 para el diagnóstico rápido de la TB y de la susceptibilidad a la RIF. ${ }^{15}$ Con este estudio se evaluó el impacto de este ensayo en los principales GV con mayor probabilidad de enfermar de TB y desarrollar resistencia.

Del total de pacientes estudiados, el $62.2 \%$ correspondió a pacientes $\mathrm{VIH}$ positivos, resultados inferiores a los reportado por Khanal y colaboradores ${ }^{16}$ que en un estudio conducido en Nepal detectaron que el $77 \%$ de los casos investigados por Xpert correspondieron a este tipo de pacientes, lo cual podría estar relacionado con que se investigaron un mayor número de pacientes que procedían de países con elevada prevalencia de TB-VIH, además, en Cuba el diagnóstico molecular de TB sólo está disponible en el IPK, por lo que la mayoría de los pacientes dentro de los GV estudiados fueron VIH positivos que procedían del Hospital de Referencia Nacional de Atención del Paciente VIH ubicado en la misma institución.

La TB es la enfermedad oportunista más común entre los pacientes infectados con el $\mathrm{VIH}$. El riesgo de un indi-
Tabla 2: Indicadores de desempeño del Xpert MTB/RIF de acuerdo con los resultados de la baciloscopia.

\begin{tabular}{|l|c|c|}
\hline $\begin{array}{c}\text { Indicadores de } \\
\text { desempeño }\end{array}$ & $\begin{array}{c}\text { Pacientes con } \\
\text { baciloscopia } \\
\text { negativa (\%) }\end{array}$ & $\begin{array}{c}\text { Pacientes con } \\
\text { baciloscopia } \\
\text { positiva (\%) }\end{array}$ \\
\hline Sensibilidad & 100.00 & 100 \\
\hline Especificidad & 92.86 & 100 \\
\hline Concordancia & 93.22 & 100 \\
\hline Valor predictivo + & 42.86 & 100 \\
\hline Valor predictivo - & 100.00 & 100 \\
\hline Índice Youden & 0.93 & 1 \\
\hline
\end{tabular}

viduo sin $\mathrm{VIH}$ de desarrollar TB puede variar de $5-15 \%$ en toda la vida, mientras que en aquellos infectados con TB y coinfectados con $\mathrm{VIH}$, el riesgo aumenta en orden de $50 \%$ en un año. ${ }^{17,18}$ La coinfección de TB-VIH sigue siendo uno de los mayores problemas de salud a nivel mundial y amenazan los programas de control de ambas enfermedades. ${ }^{19}$ La TB puede ser muy difícil de detectar en las personas seropositivas al $\mathrm{VIH}$, lo que puede retrasar el diagnóstico, por lo que la introducción del Xpert MTB/RIF es de una gran repercusión para el diagnóstico en este tipo de pacientes.

Otro de los grupos de alto riesgo de contraer TB que se estudiaron fueron los reclusos y exreclusos. En esta investigación se estudiaron cinco casos que a la vez cumplían la condición de ser VIH positivos, de los cuales en dos se detectó TB. Sobre este grupo en particular existen escasos reportes. En cualquier país las personas privadas de libertad viven con frecuencia hacinadas en instalaciones con insuficiente ventilación. Estas condiciones son propicias para el desarrollo de enfermedades infecciosas crónicas, incluyendo la TB y el $\mathrm{VIH} .{ }^{20}$ Para mitigar el riesgo de contraer TB en este grupo se hace necesario llevar a cabo urgentes intervenciones, como la reducción del hacinamiento, la búsqueda activa de sintomáticos respiratorios y el diagnóstico rápido de la enfermedad para evitar la transmisión y comenzar el tratamiento de forma rápida y oportuna. En Cuba se desconoce la prevalencia de TB en prisiones por lo que sería necesario realizar investigaciones dirigidas en especial a este grupo de riesgo para conocer la magnitud real del problema.

Nuevas tecnologías como el sistema Xpert MTB/RIF, pueden proveer la oportunidad de una temprana identificación de la TB, en menos de dos horas. El cultivo requiere más de 30 días para emitir el diagnóstico. Además, el método molecular pudiera reducir potencialmente los costos asociados con visitas a clínicas, medicamentos, radiografías de tórax, así como del tratamiento de las infecciones asociadas, con la reducción subsecuente de la morbilidad y la mor- 
Neumol Cir Torax. 2019; 78 (3): 284-289

talidad, por lo que sería muy apropiado su uso en países de ingresos bajos y medios. Es importante señalar que los métodos convencionales no deben ser reemplazados en su totalidad pues provee el perfil de resistencia no sólo a RIF, sino también a otras drogas antituberculosas. ${ }^{21,22}$

En esta investigación de los 21 casos de TB identificados por el ensayo molecular, el $71.4 \%$ se confirmó por el cultivo. El cultivo bacteriológico es la prueba de oro y desempeña un papel importante para el diagnóstico de Mtb, pero resulta muy lento y laborioso, además tiene una moderada S. ${ }^{14,23}$ Los pacientes con examen directo negativo, dependen de los resultados del cultivo para poder comenzar el tratamiento, si la carga bacteriana de la muestra es muy baja puede suceder que la micobacteria no se desarrolle en el medio de cultivo. ${ }^{14}$ El Xpert no sólo provee la ventaja de la rapidez del diagnóstico de la TB; sino que también detecta un bajo número de copias del genoma de $\mathrm{Mtb}$ en la muestra, ${ }^{24}$ por lo que el uso de este método de diagnóstico rápido sería de gran utilidad.

Resultados inferiores a los obtenidos en este trabajo fueron reportados por Loannidis y colegas $^{25}$ en Atenas, Grecia en el 2010, país de baja tasa de incidencia de TB (4.5 × 100 habitantes en el 2015), de 80 muestras pulmonares estudiadas 31 fueron positivas a Mtb por Xpert, y se confirmaron por cultivo 29 (93.5\%).

En la actual investigación, la $\mathrm{S}$ del Xpert en los casos en que la baciloscopia fue negativa, fue del 100\%. Estos resultados son superiores a lo reportado por Armand y su equipo ${ }^{26}$ que obtuvo un $57 \%$ de $S$ en 28 muestras de pacientes con baciloscopia negativa y lo reportado en el ámbito internacional (72-77\%). ${ }^{27,28}$ Estas diferencias pudieran estar dadas por el de tamaño de la muestra utilizada en esta investigación, lo cual es una limitación del estudio.

Para los pacientes con baciloscopia positiva, todos los indicadores fueron perfectos. Similares resultados obtuvieron Zeka y colaboradores ${ }^{8}$ en una investigación en Turquía realizada en 253 muestras pulmonares. Boehme y colegas $^{29}$ en un estudio multicéntrico en el 2011 reportó valores de $S$ entre 96\%-100\%, cifra que varió según los países participantes (Sudáfrica, India, Perú, Azerbaiyán, Uganda, y Filipinas).

Los resultados de la baciloscopia, al igual que el Xpert, se obtienen en el mismo día pero ésta sólo informa género, además de la $\mathrm{S}$ limitada de la técnica que para ser positiva debe tener entre 5,000 y 10,000 bacterias por mililitro de muestra. ${ }^{30}$ El beneficio de la introducción del Xpert está dado en que permitió al personal médico obtener los resultados de la detección Mtb en el mismo día y adicionalmente la información de la susceptibilidad a la RIF, comenzar rápidamente el tratamiento antituberculoso específico, lo que evitó la ocurrencia de complicaciones graves, sobre todo en los pacientes cuyo examen directo fue negativo o en personas que viven con $\mathrm{VIH}$. Por otro lado, el ensayo provee información semicuantitativa de la carga de $M t b$, que constituye un biomarcador importante para determinar la gravedad de la enfermedad y evaluar el riesgo de trasmisión. ${ }^{4,31}$

En la presente investigación sólo se identificó resistencia a RIF en una muestra, esto unido al escaso número de muestras que resultaron positivas durante el período de estudio impidió el cálculo de los ID para el Xpert en lo referente a la detección de resistencia. No obstante, es importante señalar que la resistencia identificada por el Xpert fue confirmada por el método de proporciones (MP).

En países con baja incidencia de TB, como Cuba, la prevalencia de resistencia a RIF es inferior al $5 \%$ entre los pacientes que no han sido tratados de manera previa, pero aumenta al $10 \%$ en los casos que han recibido tratamiento previo. Se recomienda en ambos casos realizar una prueba confirmatoria para establecer el diagnóstico definitivo de resistencia. Otros autores plantean que si la prevalencia de la resistencia a RIF es menor al $2 \%$ en una población, una prueba molecular positiva tiene un $50 \%$ de posibilidades de ser un error de laboratorio. ${ }^{3}$

Se concluye que el Xpert MTB/RIF permitió realizar el diagnóstico rápido de TB en un mayor número de pacientes, con un efecto muy positivo en el manejo oportuno de casos de los principales GV estudiados. La excelente correlación entre Xpert MTB/RIF y el método de las proporciones demuestra la utilidad del ensayo para la evaluación rápida de la resistencia a RIF como indicador de TB-MDR.

\section{REFERENCIAS}

1. Culqui DR, Rodríguez-Valín E, Martínez de Aragón MV. Epidemiología de las hospitalizaciones por tuberculosis en España: análisis del conjunto mínimo básico de datos 1999-2009. Enferm Infecc Microbiol Clin 2015;33(1):9-15. doi: 10.1016/j.eimc.2013.12.015.

2. Vallego VP, Rodriguez JC, Searle MA, et al. Ensayo Xpert/RIF en el diagnóstico de tuberculosis. Rev Chil Enferm Respir 2015; 31(2):127131.

3. Blakemore R, Story E, Helb D, et al. Evaluation of the analytical performance of the Xpert MTB/RIF assay. J Clin Microbiol 2010;48(7):2495-2501. doi: 10.1128/JCM.00128-10.

4. Barnard M, Gey van Pittius NC, van Helden V, Bosman M, Coetzee G, Warren RM. The diagnostic performance of the GenoType MTBDRplus version 2 line probe assay is equivalent to that of the Xpert MTB/RIF assay. J Clin Microbiol 2012;50(11):3712-3716. doi: 10.1128/JCM.01958-12.

5. Lawn SD, Mwaba P, Bates M, et al. Advances in tuberculosis diagnostics: the Xpert MTB/RIF assay and future prospects for a point-of-care test. Lancet Infect Dis 2013;13(4):349-361. doi: 10.1016/ S1473-3099(13)70008-2.

6. Steingart KR, Schiller I, Horne DJ, Pai M, Boehme CC, Dendukuri N. Xpert $\circledast$ MTB/RIF assay for pulmonary tuberculosis and rifampicin resistance in adults. Cochrane Database Syst Rev 2014;21;(1):CD009593. doi: 10.1002/14651858.CD009593.pub3.

7. Weyer K, Mirzayev F, Migliori GB, et al. Rapid molecular TB diagnosis: evidence, policy making and global implementation of Xpert MTB/RIF. Eur Respir J 2013;42(2):252-271. doi: 10.1183/09031936.00157212. 
8. Zeka AN, Tasbakan S, Cavusoglu C. Evaluation of GeneXpert MTB/RIF assay for rapid diagnosis of tuberculosis and detection of rifampicin resistance in pulmonary and extrapulmonary speciments. J Clin Microbiol 2011;49(12):4138-4141. doi: 10.1128/ JCM.05434-11.

9. Ministerio de Salud Pública. Dirección de Registros Médicos y Estadística de Salud. La Habana, Cuba: Anuario Estadístico de Salud; 2018. ISSN: 1561-4433.

10. Lemus D, Echemendía M, Díaz R, Llanes MJ, Suárez L, Marrero A. Antituberculosis drug resistance in pulmonary isolates of Mycobacterium tuberculosis, Cuba 2012-2014. MEDICC Rev 2017;19(1):10-15.

11. Resolución Ministerial No. 277. Ministerio de Salud Pública. Programa Nacional de Control de la Tuberculosis. Manual de Normas y Procedimientos. La Habana: Ciencias Médicas; 2013.

12. World Health Organization. Xpert MTB/RIF implementation manual. Technical and operational 'how-to': practical considerations, 2014. France. WHO/HTM/TB/2014.1 Fecha de consulta: septiembre, 2018. Accesible en https://www.who.int/tb/publications/xpert_implem_ manual/en/.

13. World Health Organization. Xpert MTB/RIF assay for the diagnosis of pulmonary and extrapulmonary TB in adults and children. Policy update. WHO/HTM/TB/2013.16. Fecha de consulta: agosto, 2018. Accesible en https://www.who.int/tb/publications/xpert-mtb-rif-assaydiagnosis-policy-update/en/.

14. Martínez-Romero MR, Sardiña-Aragón M, García-León G, MederosCuervo L, Vega-Riverón B, Díaz-Rodríguez R. Evaluación del sistema automatizado BacT ALERT 3D para el aislamiento de micobacterias en el LNRTB-IPK. Neumol Cir Torax 2012;71(4):333338.

15. Muñoz L, Moure R, Porta N, et al. GeneXpert@ for smear-negative pulmonary tuberculosis: does it play a role in low-burden countries? Diagn Microbiol Infect Dis 2013;75(3):325-326. doi: 10.1016/j. diagmicrobio.2012.11.011.

16. Khanal S, Baral P, Shrestha M, et al. Yield of intensified tuberculosis case-finding activities using Xpert巴 MTB/RIF among risk groups in Nepal. Public Health Action 2016;6(2):136-141. doi: 10.5588/ pha.16.0015.

17. Lozano-Salazar JL, Plasencia-Asorey CC, Costa-Montané DM, Puente-Saní V. Coinfección por tuberculosis y virus de la inmunodeficiencia humana: confluencia de dos epidemias. MEDISAN 2012;16(9):1438-1450.

18. De Paz DA, Potes L, Quiñónez E, et al.; Grupo de Investigación de Tuberculosis de la Fundación Valle del Lili. Resistencia a fármacos antituberculosis en pacientes coinfectados con tuberculosis y virus de la inmunodeficiencia humana, en un hospital de referencia de 2007 a 2010 en Cali, Colombia. Infectio 2012;16(3):161-165. doi: 10.1016/ S0123-9392(12)70006-1.

19. Peralta-Gómez I, Cabrera-Rodríguez MC, Gutiérrez-Díaz MJ. Coinfección TB/VIH: una amenaza para los programas de control de ambas enfermedades. Medicent Electrón 2015;19(3):160-162.
20. Zarate E, Lobón I, Saavedra C, Castañeda M. Tuberculosis en nuevos escenarios: establecimientos penitenciarios. An Fac Med Lima 2005;66(2):148-158.

21. Iram S, Zeenat A, Hussain S, Wasim Yusuf N, Aslam M. Rapid diagnosis of tuberculosis using Xpert MTB/RIF assay- report from a developing country. Pak J Med Sci 2015;31(1):105-110. doi: 10.12669/ pjms.311.6970.

22. Peñata A, Salazar R, Castaño T, Bustamante J, Ospina S. Diagnóstico molecular de tuberculosis extrapulmonar y sensibilidad a rifampicina con un método automatizado en tiempo real. Biomédica 2016;36(Supl.1):78-89. doi: https://doi.org/10.7705/biomedica. v36i3.3088.

23. Bajrami R, Mulliqi G, Kurti A, Lila G, Raka L. Comparison of GeneXpert MTB/RIF and conventional methods for the diagnosis of tuberculosis in Kosovo. J Infect Dev Ctries 2016;10(4):418-422. doi: 10.3855/iidc.7569.

24. Agrawal M, Bajaj A, Bhatia V, Dutt S. Comparative study of GeneXpert with ZN stain and culture in samples of suspected pulmonary tuberculosis. J Clin Diagn Res 2016;10(5):DC09-12. doi: 10.7860/ JCDR/2016/18837.7755.

25. Ioannidis P, Papaventsis D, Karabela S, et al. Cepheid GeneXpert MTB/RIF assay for Mycobacterium tuberculosis detection and rifampin resistance identification in patients with substantial clinical indications of tuberculosis and smear-negative microscopy results. J Clin Microbiol 2011;49(8):3068-3070. doi: 10.1128/JCM.00718-11.

26. Armand S, Vanhuls P, Delcroix G, Courcol R, Lemaître N. Comparison of the Xpert MTB/RIF test with an IS6110-TagMan real-time PCR assay for direct detection of Mycobacterium tuberculosis in respiratory and nonrespiratory specimens. J Clin Microbiol 2011;49(5):1772-1776. doi: 10.1128/JCM.02157-10.

27. Marlowe EM, Novak-Weekley SM, Cumpio J, et al. Evaluation of the Cepheid Xpert MTB/RIF assay for direct detection of Mycobacterium tuberculosis complex in respiratory specimens. J Clin Microbiol 2011:49(4):1621-1623. doi: 10.1128/JCM.02214-10.

28. Moure R, Muñoz L, Torres M, Santin M, Martín R, Alcaide F. Rapid detection of Mycobacterium tuberculosis complex and rifampin resistance in smear-negative clinical samples by use of an integrated real-time PCR method. J Clin Microbiol 2011;49(3):1137-1139. doi: 10.1128/JCM.01831-10.

29. Boehme CC, Nicol MP, Nabeta P, et al. Feasibility, diagnostic accuracy, and effectiveness of decentralised use of the Xpert MTB/RIF test for diagnosis of tuberculosis and multidrug resistance: a multicenter implementation study. Lancet 2011;377(9776):1495-1505. doi: 10.1016/ S0140-6736(11)60438-8.

30. Organización Panamericana de la Salud. Manual para el Diagnóstico Bacteriológico de la Tuberculosis. Parte 1. Manual de actualización de la baciloscopia, 2018.

31. Arias MF, Herrera MT. Nuevos métodos para el diagnóstico de la tuberculosis. Rev Chil Enferm Respir 2016;32(4):254-259.

Conflicto de intereses: Los autores declaran no tener conflicto de intereses. 